\title{
Genome-wide analysis of immunophilin FKBP genes and expression patterns in Zea mays
}

\author{
W.-W. Wang ${ }^{1,2}$, Q. $\mathrm{Ma}^{2}$, Y. Xiang ${ }^{2}$, S.-W. Zhu' ${ }^{2}$ and B.-J. Cheng ${ }^{2}$ \\ ${ }^{1}$ School of Chemistry and Life Sciences, Suzhou University, Suzhou, \\ Anhui, China \\ ${ }^{2}$ Anhui Province Key Lab of Crop Biology, School of Life Sciences, \\ Anhui Agricultural University, Hefei, Anhui, China \\ Corresponding author: B.-J. Cheng \\ E-mail: beijiucheng@ahau.edu.cn
}

Genet. Mol. Res. 11 (2): 1690-1700 (2012)

Received November 25, 2011

Accepted April 18, 2012

Published June 25, 2012

DOI http://dx.doi.org/10.4238/2012.June.25.2

\begin{abstract}
The receptors for the immunosuppression drugs FK506 and rapamycin are called FKBPs (FK506-binding proteins). FKBPs comprise a large family; they are found in many species, including bacteria, fungi, animals, and plants. As a class of peptidyl-prolyl cistrans isomerase enzymes, the FKBP genes have been the focus of recent studies on plant stress tolerance and immunology. We identified and analyzed gene families encoding these proteins in maize using computational and molecular biology approaches. Thirty genes were found to encode putative FKBPS according to their FK506-binding domain. The $F K B P$ genes can be classified into single domain and multiple domain members based on the number of the domains. By analysis of the physical locations, the $30 \mathrm{FKBP}$ genes were found to be widely distributed on 10 chromosomes. After analysis of the FKBP phylogenetic tree in the maize genome, we found that the 30 genes revealed two major clades. Gene duplication played a major role in the evolution of $F K B P$ genes, which suggests that the $F K B P$ genes in maize have a pattern significantly different from that of these genes in rice. Based on semi-quantitative RT-PCR, we found that the 30 FKBPs were expressed differently in various tissues in maize, which suggests
\end{abstract}


that $F K B P$ genes play different roles in each tissue. Several FKBPS were expressed at higher levels in roots, indicating that these genes in maize may have similar or overlapping functions.

Key words: Maize; FKBPs; Gene duplication; Expression

\section{INTRODUCTION}

FK506 is a macrolide immunosuppressant composed of 23 rings. Proteins that can bind it are called FK506-binding proteins (FKBPs). This is a class of highly homologous receptor-binding proteins that are widely present in all organisms, including bacteria, fungi, animals, and plants. FKBPs are mainly divided according to molecular weight difference, such as FKBP12, FKBP15, FKBP22, FKBP55, and so on. As cellular receptors for the immunosuppressant drug ligands FK506 and rapamycin (Siekierka et al., 1989; Harding et al., 1989), FKBPs can catalyze the cis to trans configuration of the N-terminal peptide bond of proline residues in peptide or protein substrates, and consequently the FKBPs have peptidyl-prolyl cis-trans isomerase (PPIase) activity (Fischer et al., 1989; Harding et al., 1989), which may be involved in protein folding processes (Brillantes et al., 1994). In many plants, FKBP genes have been found to be vital for regulating normal growth and development, for coping with stress conditions and fundamental considerations for modern cereal farming. Other findings suggest that $F K B P$ genes can operate as molecular chaperones (Hartl et al., 2002). All of these points suggest that these proteins participate in important cellular processes. Consequently, $F K B P$ genes have been the focus in plant-related subject areas.

FKBPs have been characterized by the FKBP signature residues that form the binding pocket for FK506 or rapamycin (Schreiber, 1991), and the FKBP signature is derived from the available structures of the FKBP12 protein (Michnick et al., 1991; Van Duyne et al., 1991). It was found that the residues required for binding the drug are highly conserved, and the conserved binding site is therefore used as the signature for the FKBP protein identification, which is called the FK506-binding domain (FKBd). Structure analysis of FKBPs has shown that they are composed of at least one FKBd, which forms a highly conserved structure (Galat, 2000). The size of the FKBPs vary significantly, ranging from single-domain (SD) isoforms comprising a single FKBd, to large (>100 kDa) complex multi-domain (MD) proteins (Galat, 2003; Rulten et al., 2006). The most typical SD isoform is FKBP12, which only has 110 amino acids. In maize, FKBP12 binds rapamycin (Agredano-Moreno et al., 2007), forming a complex that inhibits the "target of rapamycin" (TOR) kinase, which can powerfully regulate the germination and the development of the plant (Marivet et al., 1995). Every FKBP has a high sensitivity for different substrates with differences up to a thousand times, showing substrate selectivity. These features imply that PPIase activity of FKBPs plays an important role in the cell. Recently, many studies have begun to examine the function of FKBPs in plants. For example, the disruption of AtFKBP42 gene function causes developmental defect (Kamphausen et al., 2002). Another study has shown that a chloroplast FKBP can interact with a photosynthetic electron carrier, which affects the accumulation of the protein (Gupta et al., 2002). As in animals, different members of FKBPs appear to play different roles in plants.

In recent years, studies have carried out several valuable functional characterizations of individual plant FKBPs, but the identification and analyses of all FKBPs have only been in 
Arabidopsis (He et al., 2004; Romano et al., 2005) and Oryza sativa (rice) (Gollan and Bhave, 2010) genomes, which have revealed the true size and character of the FKBP family in higher plants. Bioinformatics have opened up new and highly efficient analysis and research approaches (Staskawicz et al., 1995), and can be used to conduct a comprehensive and complete analysis of the basic data of the whole genome with regard to FKBP genes, which play an important role in large-scale gene isolation, gene functional annotation and functional genomics studies. Zea mays L. (maize) is the most widely cultivated plant in the world, which also provides a model genome for molecular study. Therefore, we took advantage of the sequenced genome of the Zea mays L. (maize) and selected bioinformatics applications to perform a genome analysis of the entire FKBP family of maize.

\section{MATERIAL AND METHODS}

\section{The genome of maize}

In this study, the complete genome sequence of maize was searched in the maize genome website (http://www.maizesequence.org/index.html).

\section{Identification of $F K B P$ genes and sequence analysis}

The key word "fkbp" was used to search for the FKBP genes in NCBI, and through the Pfam database, we then obtained the conserved sequence of the FKBd-c domain. The Hidden Markov model profile of the FKBd-c domain was used as a query in BLASTP searches for possible homologs in maize genome databases. The threshold expectation value was set to $10^{-3}$, a value determined empirically to filter out most of the spurious hits. This step was crucial to find the maximum number of candidate genes. By using the Pfam database (Protein family) (http://pfam.janelia.org), we analyzed all the candidate gene sequences to remove genes that did not contain a FKBd-c domain. We then aligned the sequences using the ClustalW software (Thompson et al., 1994).

The molecular weights and isoelectric points (pI) of putative FKBPs were predicted using the Compute pI/MW Tool at the Expert Protein Analysis System (ExPAsy) site (http:// au.expasy.org/tools/pi_tool.html).

\section{Physical locations of $F K B P$ genes}

The physical locations of all regular FKBP genes were confirmed by tBLASTn (P value $=0.001$ ) search using a local database of complete Zea mays L. genome sequences of each chromosome. Afterwards, the Genome Pixelizer software (http://www.niblrrs.ucdavis. edu/GenomePixelizer/GenomePixelizer_Welcome.html) was used for a graphic portrayal of FKBP genes of maize.

\section{Phylogenetic tree construction}

Multiple amino acid sequence alignments of the maize $F K B P$ genes were performed using the ClustalW software. In order to analyze the evolutionary relationship of the FKBP 
genes in maize, we constructed the phylogenetic tree using the bootstrap neighbor-joining (NJ) method (1000 bootstrap replicates) with a Kimura 2-parameter model by MEGA 3.1 (http://www.megasoftware.net/).

In order to analyze the evolutionary relationships of the $F K B P$ genes between different plants, we compared the FKBPS in maize and rice. We obtained the $29 F K B P$ sequences of rice according to Gollan and Bhave (2010). Along with the 30 FKBPS in maize, there were 59 FKBPs in all for construction of the phylogenetic tree. Multiple alignments of 59 amino acid sequences were performed using ClustalX with default options. Phylogenetic trees were constructed based on the alignment by Clustalx1.81.

\section{Semi-quantitative RT-PCR analysis}

The maize seeds of B73 were germinated on plastic plates, and the plants were then transferred to a nutrient solution at $28^{\circ} \mathrm{C}$ in a culture room under a 16 -h light and 8 -h dark photoperiod.

Total RNAs were extracted from various tissues (leaves, stems and roots) using the RNAiso reagent (Tiandz Biotechnology) according to manufacturer instructions. Firststrand cDNAs were generated from the RNA with the Access RT-PCR system (Promega, USA). The gene-specific primers used for ZmFKBPs were designed to examine the expression patterns of $F K B P$ genes. The expression of the actin gene was used as an internal control to normalize the variation in the quality of RNA between different reactions. PCR was performed using Taq polymerase (Tiandz Biotechnology) on a thermal cycler (Tpersonal 48; Biometra, Germany). The RT-PCRs were repeated three times and consistent results were obtained.

\section{RESULTS}

\section{Identification and nomenclature of $F K B P$ genes in maize}

In order to identify the $F K B P$ genes, we checked the sequenced genome of maize for these genes using the multiple methods described above; 44 FKBP genes were identified in the maize genome. After removal of overlapping sequences, we finally obtained 30 distinct loci encoding putative $F K B P$ s containing the characteristic FKBd (Table 1). These genes were then analyzed using the Pfam database; the results showed that the 30 genes all contained highly conserved FKBds. Finally, we checked the FKBds in each of the gene sequences manually. FKBd analysis categorized these FKBPs into single and multiple domain genes.

In this study, the nomenclature also adopted the rules that are rather consistent in previously published $F K B P S$ in animals and plants. The proteins are designated $F K B P$ with the prefix letters "Zm" and a suffix number. The suffix numbers are labeled according to their orthology with reported isoforms in Oryza sativa (rice), rather than solely based on their estimated molecular weights. In some cases, the similarities in domain structures and pI were also considered, for example, the putative FKBP denoted as 'ZmFKBP13' from maize showing the highest amino acid identity with OsFKBP13, despite its predicted molecular mass being $22 \mathrm{kDa}$ (Table 1). 


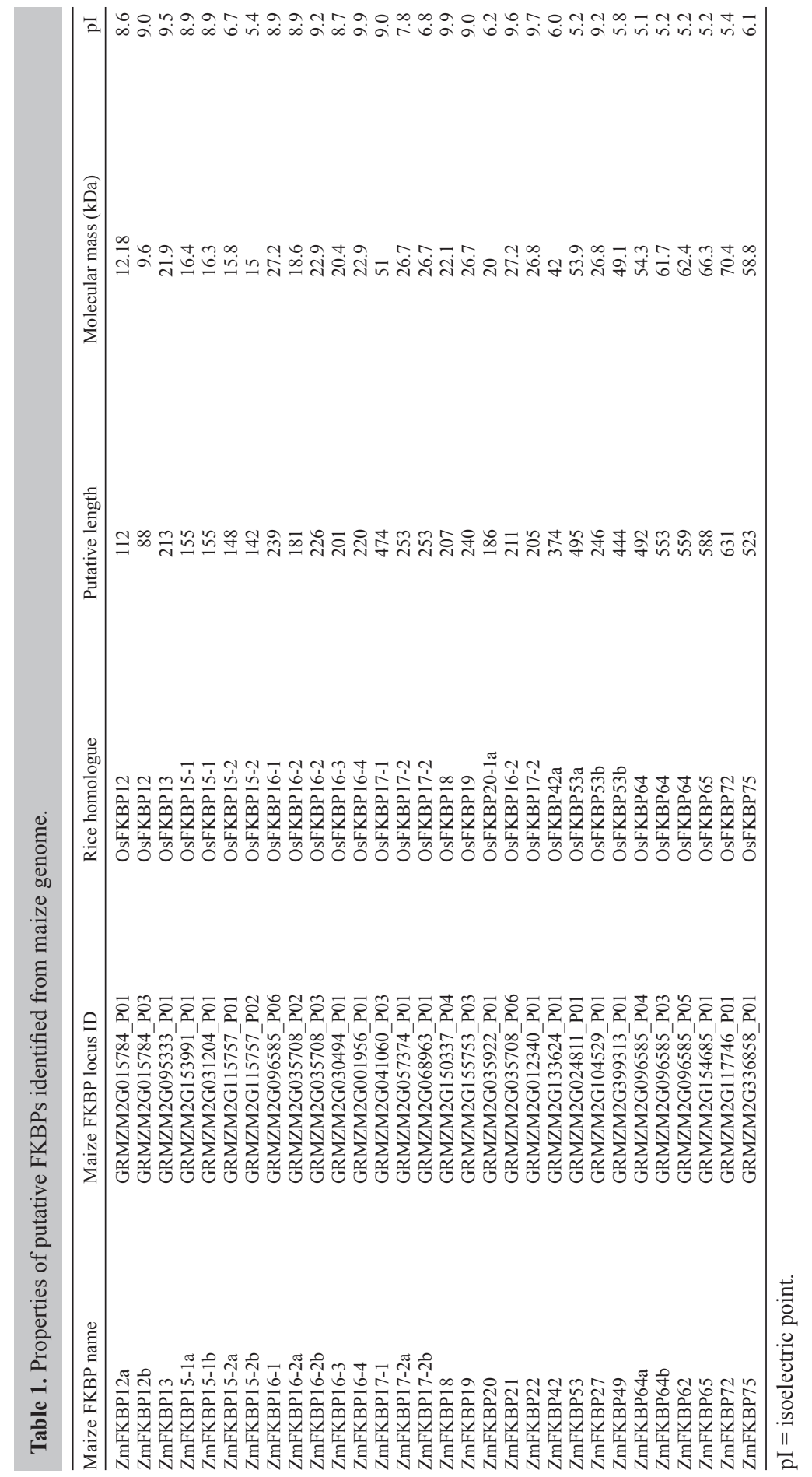




\section{Chromosomal locations and genomic duplications of maize FKBP genes}

In maize, $F K B P$ genes were widely distributed on 10 chromosomes from chromosome 1 to chromosome 10 (Figure 1). Chromosomes 1 and 5 had the largest number of $F K B P$ genes (7), and chromosome 7 had 4 FKBP genes. On chromosomes 2 and 4, there were 3 FKBP genes, respectively, chromosome 9 had 2, and the rest only had 1. According to the definition of gene cluster, there are three or more genes in the same chromosome and the neighboring regions are less than $200 \mathrm{~kb}$ (Holub, 2001). From the physical location map (Figure 1), there are 14 genes in 3 gene clusters, and other genes were rather equally distributed on each chromosome. According to He et al. (2004), the gene cluster of the FKBPs in Arabidopsis is not obvious, which suggests that maize showed a significantly different pattern from Arabidopsis and other plants. In the evolution of maize, the FKBP gene may have arisen from gene duplication and recombination.

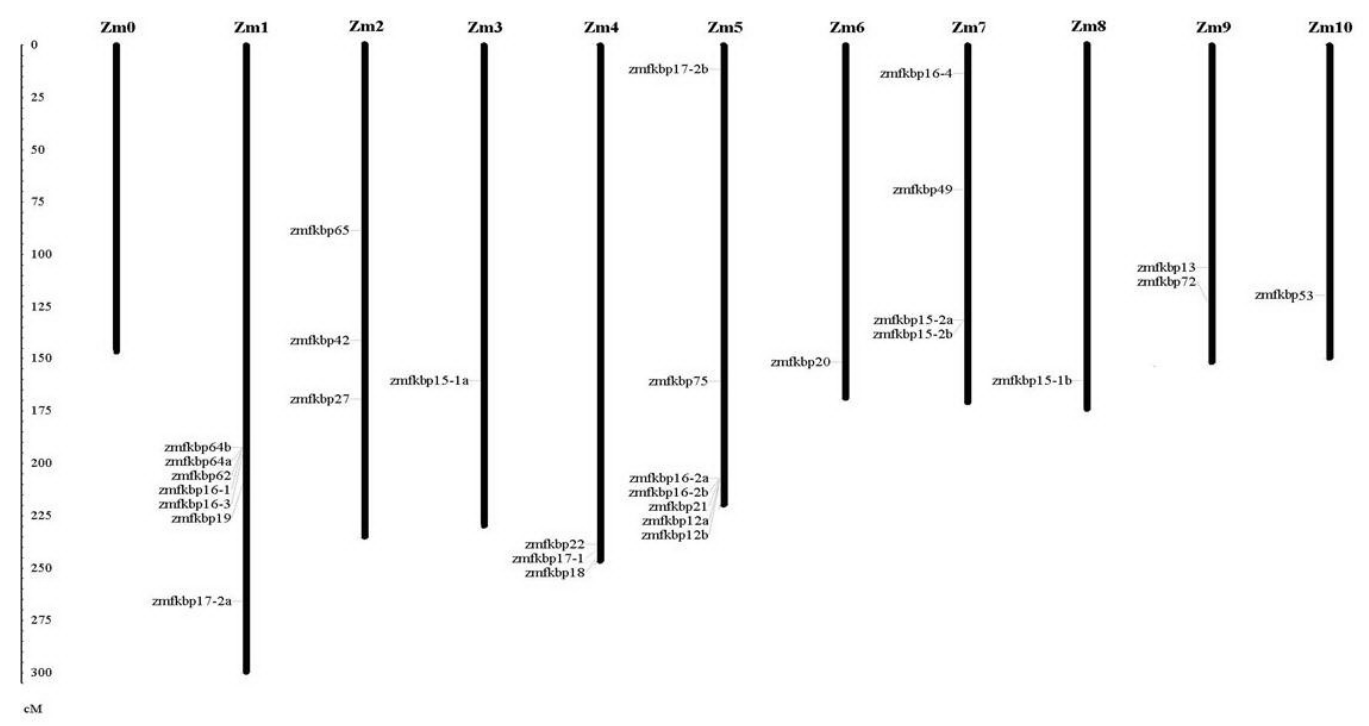

Figure 1. Physical locations of $F K B P$ genes in maize.

According to the definition of gene duplication: 1) in a relatively long genome, more than $70 \%$ of the nucleotide sequence is arranged, and 2) the similarity of the nucleotide sequence in the arranged area is more than $70 \%$ (Zhou et al., 2004). In maize, there were 18 genes produced by gene duplication, which accounted for $60 \%$ of the total ZmFKBPs. In addition, one ZmFKBP possibly resembled several other ZmFKBPs, for example, ZmFKBP62 was gene duplicated from ZmFKBP64a, ZmFKBP64b and ZmFKBP16-1. This showed that most of the ZmFKBPs were generated from gene duplication and that large-scale gene duplication occurred during genome evolution. The results showed that gene duplication plays an important role in the quantitative expansion of the FKBP genes. 


\section{Phylogenetic analysis of the FKBP genes in maize}

To analyze phylogenetic relationships of the $F K B P$ genes among maize, phylogenetic tree were constructed based on the alignments of ZmFKBP protein sequences (Figure 2). Because of the highly conserved amino acid sequences of the FKBP genes and the region containing similar motifs, the neighbor-joining method of the MEGA 3.1 software was adopted to construct the phylogenetic trees for the 30 FKBPs in maize.

From the phylogenetic tree (Figure 2), the FKBP genes in maize showed high sequence similarity between the members of the all family. The tree showed the FKBP genes distributed in two distinct clades, and the members in each clade various showed more significant similarity. In addition, the overall sequence similarity between the members showed some differences. Most of the FKBP genes had a near relationship, tending to cluster in one branch, but also several genes had a relatively distant relationship and distributed in one branch (Figure 2), which suggests that the evolution of the FKBP genes in maize is complex.

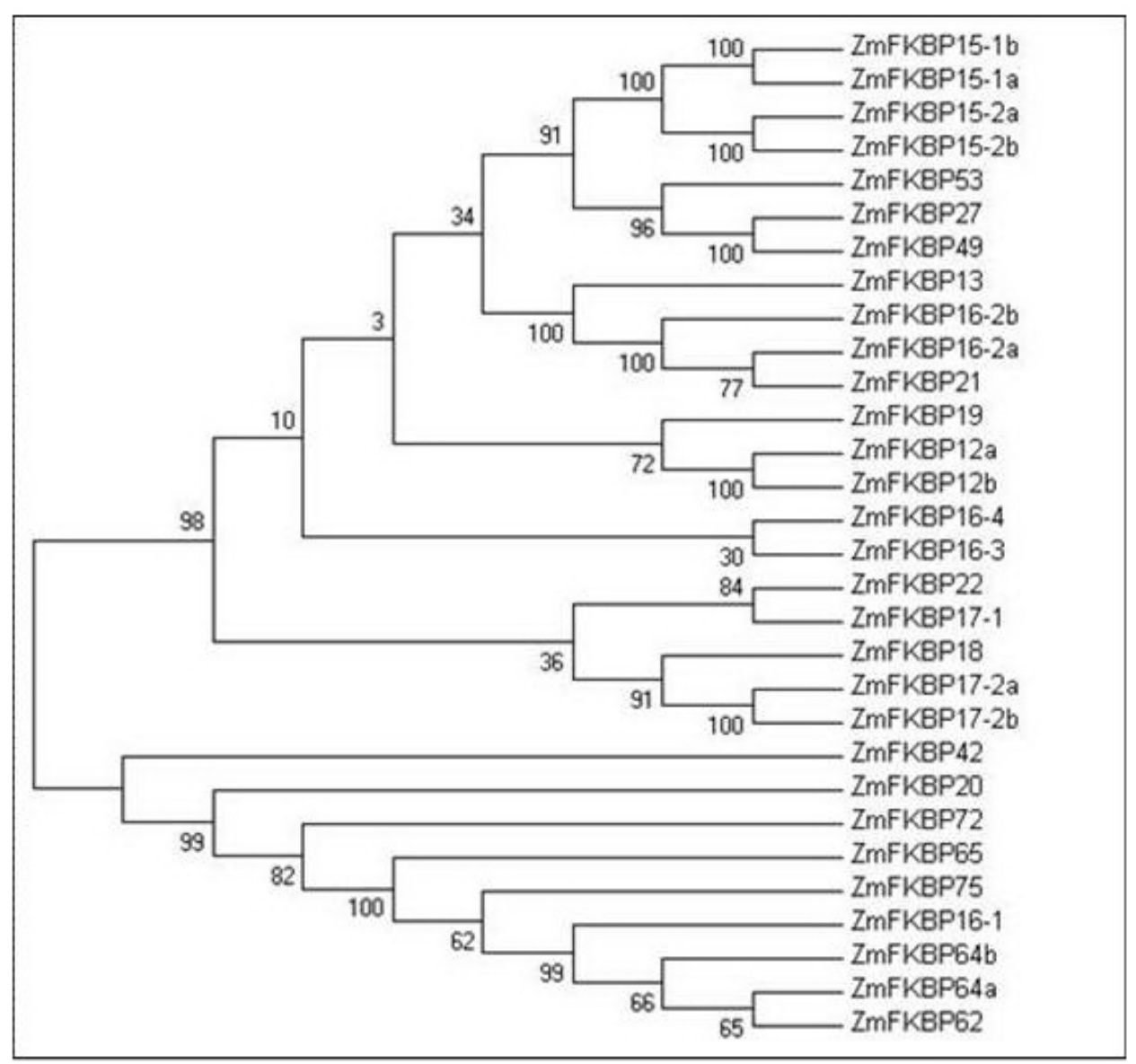

Figure 2. Phylogenetic analyses of the $F K B P$ genes in the maize genome. 


\section{Evolutionary analysis comparative of the FKBP genes between maize and rice}

In order to analyze the evolutionary relationships of the $F K B P$ genes in different species, the FKBPs in maize and rice were compared, and the phylogenetic tree was constructed based on the alignments of ZmFKBP and OsFKBP protein sequences. The tree shows multiple smaller branches but few big branches, which was very different from maize. From the tree (Figure 3), we can see not only the contact between maize and the rice, but also an obvious difference. For almost every FKBP gene in rice, there was one or more corresponding ZmFKBPs showing the highest sequence similarity (Figure 3), thereby indicating that the $F K B P s$ in maize and rice have high homology. In maize, the number of the FKBPs is more than in rice, so it appears that several ZmFKBPs correspond to other ZmFKBPs, but not OsFKBPs, and this may be due to the gene duplication in maize. This also indicates that the evolution of the FKBPs in maize is more complex.

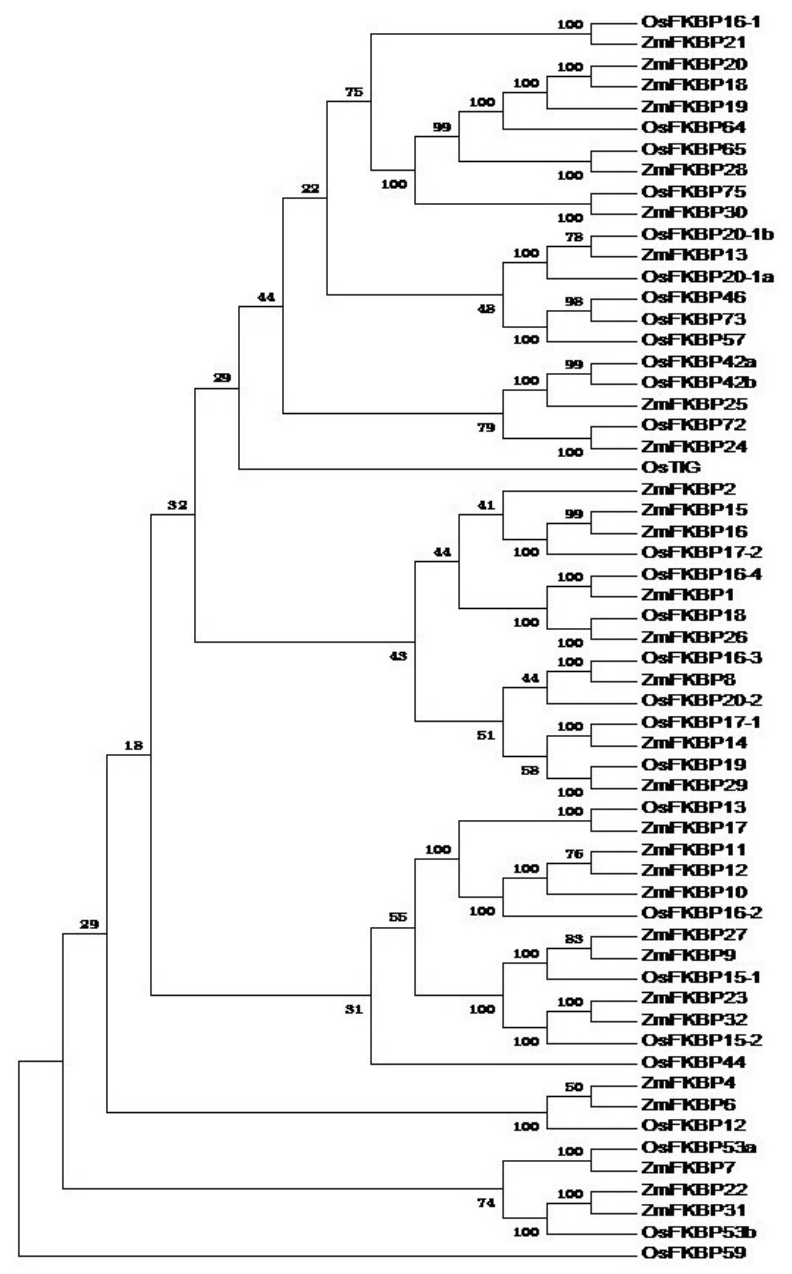

Figure 3. Phylogenetic analyses of the FKBP genes in the maize and rice genomes. 


\section{Expression of $F K B P$ genes}

The expression of the FKBP genes was examined by RT-PCR. RT-PCR analysis indicated that all 30 FKBP genes were expressed in maize plants. The expression patterns of the genes are shown in Figure 4. The genes were expressed in different tissues including roots, stems and leaves, but expression was detected at different levels. From the figure we can clearly see the different expression patterns; the expression of several genes was higher in both leaves and stems compared to roots, such as ZmFKBP18 and ZmFKBP19. There were some special genes that only expressed in one or two tissues and not expressed in the second or third tissue. A special gene, ZmFKBP17-2a, appeared to be only expressed in roots, implicating ZmFKBP17-2a in further specific functions. Moreover, for some close relationship genes, they showed complementary gene expression patterns, such as ZmFKBP64a and ZmFKBP64b. All of the results suggest that the 30 FKBP genes are expressed in every tissue of maize but at different levels. This expression pattern provides an important basis for further study of the roles and function of the FKBP genes.

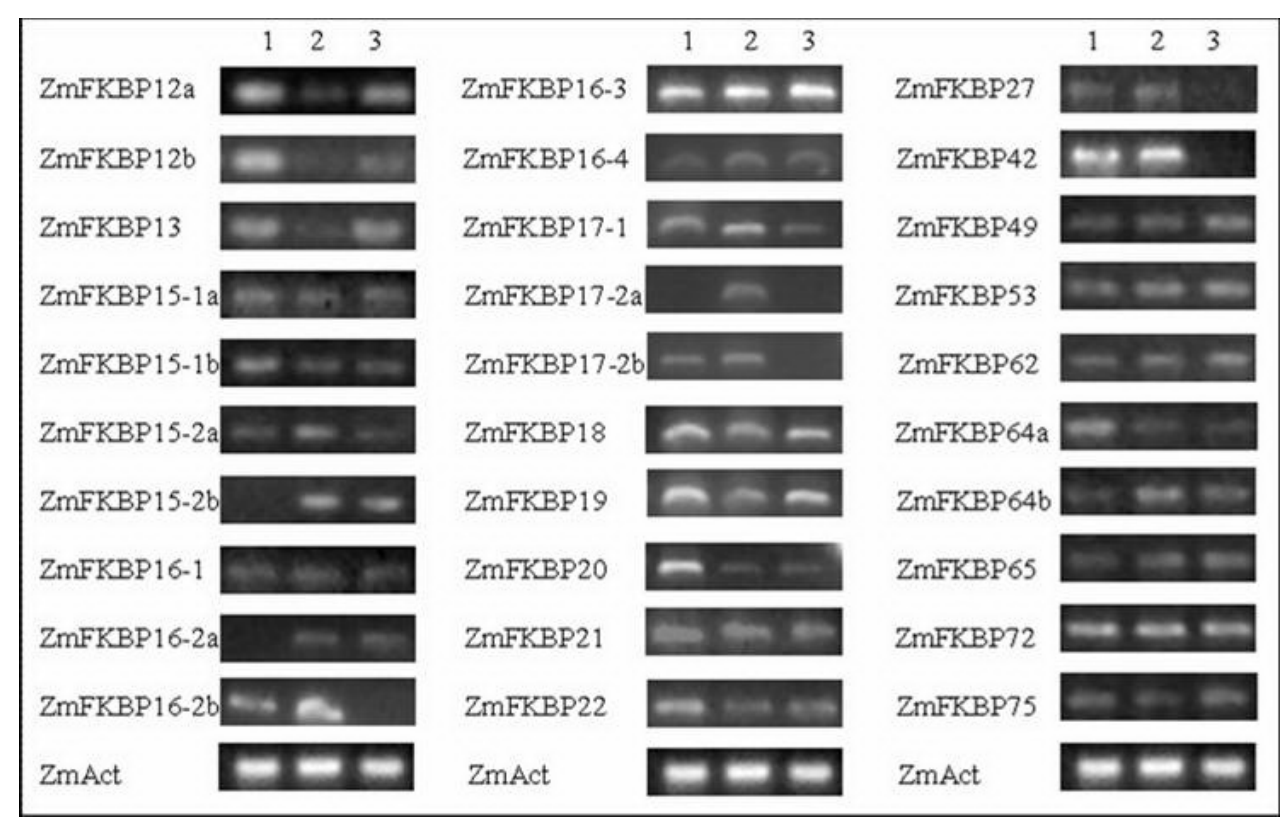

Figure 4. Electrophoresis of $F K B P$ RT-PCR products. Lane $1=F K B P$ gene in leaves; lane $2=F K B P$ gene in roots; lane $3=F K B P$ gene in stems, and $\mathrm{ZmAct}$ was used as a template control.

\section{DISCUSSION}

The FK506- and rapamycin-binding proteins are abbreviated as FKBPs. FKBPs have been identified in all organisms examined, including bacteria, fungi, animals, and plants (Schreiber, 1991; Fruman et al., 1994; Luan, 1998), and they have been extensively studied and described in other species. In this study, we analyzed the sequence of the Zea mays L. (maize) 
genome and revealed 30 FKBP genes, which comprise the largest FKBP family so far reported. For example, the yeast genome contains 4 genes for FKBPS (Heitman et al., 1991), and the human genome contains 18 FKBPs (Uchida et al., 1999). The large number of genes for immunophilins in plants indicates a diverse array of functions served by these proteins, while this may also reflect a significant degree of functional redundancy among the genes. Among plants, maize also contains a larger number than others, where this may be due to the frequency of the $F K B P$ gene duplications in maize. The origin of FKBP genes, formation of gene families and the multiplicity of gene expression can be understood by analyzing gene duplication mechanisms. In maize, gene duplication analysis shows that at least 18 FKBPs occurring as duplicates in 30 maize FKBP families, the ratio of gene duplications was very high. However, in Arabidopsis, the studies reported there were only 4 duplicated genes of the 23 AtFKBPs (He et al., 2004), which were less than the maize. Not only the numbers but also the gene duplications in maize were all significantly increased compared to other plants. Therefore, the evolutionary patterns in Zea mays L. were special, which was likely the result of gene duplication events.

Immunophilins consist of a very diverse group of proteins and may have very different origins during genome evolution. Studies in maize report an uneven chromosomal distribution of $F K B P$ genes, and the majority of the genes have been found in clusters, which may be due to the high frequency of gene duplication. The FKBP genes of maize were widely distributed on 10 chromosomes, and the distribution showed great differences, where chromosome 1 and 5 contained the maximum number, which revealed the important role of the two chromosomes in the evolution of FKBP genes. In the maize 30 FKBP genes, $60 \%$ fell in clusters; however, there were no obvious clusters in the Arabidopsis genome. This result indicated that gene duplication played an important role in gene expansion.

Based on the analysis of the phylogenetic trees of the FKBP genes, the maize sequences showed two major clades, which was different from rice. Rice sequences exhibited no notable clustering, rather giving rise to several small branches, which may due to its different structure. Furthermore, the gene had high homology, often distributed in one branch, which was very similar to maize. Therefore, we infer that the two monocotyledons have similarities in evolution, and that they may have an analogous mode of selection and evolution. Despite strong homology, the number of the genes and the chromosomal locations in maize are different compared to rice. These differences may reflect different kinds of ancient duplication, which also demonstrate that FKBPs in maize comprise an extraordinary family.

The transcript levels were much lower in roots as compared to those in the green tissues (leaves and stems) in Arabidopsis, according to Luan et al. (2004). In our study, FKBPs expressed in every tissue of maize, but their expression does not follow obvious rules, which means every tissue has its strongest expressed gene in maize. This result may be due to different environmental factors and different tissues used for the expression of these genes in this study. We found that ZmFKBP16-2b, ZmFKBP16-3 and ZmFKBP42 were all expressed higher in roots, indicating that these three genes in maize may have similar or overlapping functions. The data presented here indicate that the expression intensity of the $30 \mathrm{FKBP}$ genes was different in every tissue, so every $F K B P$ plays different roles in each tissue.

\section{ACKNOWLEDGMENTS}

Research supported by the Key Projects in the National Science \& Technology Pillar 
Program (\#2009BADA6B00), the National Natural Science Foundation (\#11075001) and the Master Scientific Research Foundation of Suzhou University (\#2011yss02).

\section{REFERENCES}

Agredano-Moreno LT, Reyes dlC, Martinez-Castilla LP and Sanchez de JE (2007). Distinctive expression and functional regulation of the maize (Zea mays L.) TOR kinase ortholog. Mol. Biosyst. 3: 794-802.

Brillantes AB, Ondrias K, Scott A, Kobrinsky E, et al. (1994). Stabilization of calcium release channel (ryanodine receptor) function by FK506-binding protein. Cell 77: 513-523.

Fischer G, Wittmann-Liebold B, Lang K, Kiefhaber T, et al. (1989). Cyclophilin and peptidyl-prolyl cis-trans isomerase are probably identical proteins. Nature 337: 476-478.

Fruman DA, Burakoff SJ and Bierer BE (1994). Immunophilins in protein folding and immunosuppression. FASEB J. 8: 391-400.

Galat A (2000). Sequence diversification of the FK506-binding proteins in several different genomes. Eur. J. Biochem. 267: 4945-4959.

Galat A (2003). Peptidylprolyl cis/trans isomerases (immunophilins): biological diversity-targets-functions. Curr. Top. Med. Chem. 3: 1315-1347.

Gollan PJ and Bhave M (2010). Genome-wide analysis of genes encoding FK506-binding proteins in rice. Plant Mol. Biol. 72: 1-16.

Gupta R, Mould RM, He Z and Luan S (2002). A chloroplast FKBP interacts with and affects the accumulation of Rieske subunit of cytochrome bf complex. Proc. Natl. Acad. Sci. U. S. A. 99: 15806-15811.

Harding MW, Galat A, Uehling DE and Schreiber SL (1989). A receptor for the immunosuppressant FK506 is a cis-trans peptidyl-prolyl isomerase. Nature 341: 758-760.

Hartl FU and Hayer-Hartl M (2002). Molecular chaperones in the cytosol: from nascent chain to folded protein. Science 295: 1852-1858.

He Z, Li L and Luan S (2004). Immunophilins and parvulins. Superfamily of peptidyl prolyl isomerases in Arabidopsis. Plant Physiol. 134: 1248-1267.

Heitman J, Movva NR and Hall MN (1991). Targets for cell cycle arrest by the immunosuppressant rapamycin in yeast. Science 253: 905-909.

Holub EB (2001). The arms race is ancient history in Arabidopsis, the wildflower. Nat. Rev. Genet. 2: 516-527.

Kamphausen T, Fanghanel J, Neumann D, Schulz B, et al. (2002). Characterization of Arabidopsis thaliana AtFKBP42 that is membrane-bound and interacts with Hsp90. Plant J. 32: 263-276.

Luan S (1998). Immunophilins in animals and higher plants. Bot. Bull. Acad. Sin. 39: 217-223.

Marivet J, Frendo P and Burkard G (1995). DNA sequence analysis of a cyclophilin gene from maize: developmental expression and regulation by salicylic acid. Mol. Gen. Genet. 247: 222-228.

Michnick SW, Rosen MK, Wandless TJ, Karplus M, et al. (1991). Solution structure of FKBP, a rotamase enzyme and receptor for FK506 and rapamycin. Science 252: 836-839.

Romano P, Gray J, Horton P and Luan S (2005). Plant immunophilins: functional versatility beyond protein maturation. New Phytol. 166: 753-769.

Rulten SL, Kinloch RA, Tateossian H, Robinson C, et al. (2006). The human FK506-binding proteins: characterization of human FKBP19. Mamm. Genome 17: 322-331.

Schreiber SL (1991). Chemistry and biology of the immunophilins and their immunosuppressive ligands. Science 251: 283-287.

Staskawicz BJ, Ausubel FM, Baker BJ, Ellis JG, et al. (1995). Molecular genetics of plant disease resistance. Science 268: 661-667.

Thompson JD, Higgins DG and Gibson TJ (1994). CLUSTAL W: improving the sensitivity of progressive multiple sequence alignment through sequence weighting, position-specific gap penalties and weight matrix choice. Nucleic Acids Res. 22: 4673-4680.

Uchida T, Fujimori F, Tradler T, Fischer G, et al. (1999). Identification and characterization of a $14 \mathrm{kDa}$ human protein as a novel parvulin-like peptidyl prolyl cis/trans isomerase. FEBS Lett. 446: 278-282.

Van Duyne GD, Standaert RF, Karplus PA, Schreiber SL, et al. (1991). Atomic structure of FKBP-FK506, an immunophilin-immunosuppressant complex. Science 252: 839-842.

Zhou T, Wang Y, Chen JQ, Araki H, et al. (2004). Genome-wide identification of NBS genes in japonica rice reveals significant expansion of divergent non-TIR NBS-LRR genes. Mol. Genet. Genomics 271: 402-415. 